\title{
VALIDATION OF LIDAR CALIBRATION USING A LIDAR SIMULATOR
}

\author{
Gyuseok Lee ${ }^{1}$, Jangwoo Cheon ${ }^{1}$, Impyeong Lee ${ }^{1}$ \\ ${ }^{1}$ Dept. of Geoinformatics, University of Seoul, Seoul, Republic of Korea - (ys96000, khai0614, iplee)@uos.ac.kr
}

Commission I, WG I/2

KEY WORDS: LIDAR, Calibration, LIDAR Simulator, Intrinsic Parameters, Plane-Based Calibration, Tilted Scan

\begin{abstract}
:
LIDAR is being widely used for mapping and modelling because it accurately scans and acquires 3D geometric information of the surrounding environment. In order to improve the accuracy of the LIDAR measurement, it is necessary to precisely estimate the intrinsic parameters as well as extrinsic parameters and eliminate the systematic errors. Many studies are conducted to eliminate these errors caused by the intrinsic parameters of LIDAR. However, when the result of intrinsic calibration is verified using actual LIDAR data, there is a problem that other error factors cannot be excluded. Therefore, in this study, the LIDAR intrinsic calibration is verified by using a LIDAR simulator that simulates the mechanism of the actual LIDAR. When constructing a LIDAR simulator, the systematic error is inserted according to the intrinsic parameter model of LIDAR. And according to the method of scanning with LIDAR, it is divided into upright scanning and tilted scanning, and the error included LIDAR simulation data is generated. After that, the intrinsic parameters are estimated by applying the plane-based intrinsic calibration. Since values of the intrinsic parameters are known, they are compared with the estimated parameters, and the results of estimate are analyzed according to the scanning method.
\end{abstract}

\section{INTRODUCTION}

The LIDAR is a sensor that fires a laser pulse in the designed direction to observe the distance and calculate the exact 3D position of the reflected point. In order to build a precise DEM data, a LIDAR is mounted on an aircraft and used to scan the ground. Recently, it has been used as a sensor for $3 \mathrm{D}$ recognition and decision of autonomous vehicles. In addition, a LIDAR is being used for the purpose of constructing a precise map through precise mapping to the surrounding environment. Sometimes, it is assumed that there is no error in the data obtained with the LIDAR, but the actual LIDAR data contains various errors. The errors that can be included in the LIDAR are intrinsic parameters such as the position and attitude of the LIDAR, intrinsic parameters such as offsets in the laser installed inside the LIDAR, and random error in distance measurement. Many studies to eliminate these errors have been conducted. (Muhammad, Lacroix, 2010) and (Skaloud, Lichti, 2006).

In order to remove the errors and improve accuracy, it is important to eliminate the systematic error of the LIDAR through intrinsic calibration, but in the case of a LIDAR, the error cannot be removed through reverse measurement like a total station. Therefore, intrinsic calibration studies such as point-based calibration and plane-based calibration have been conducted. (Chow et al., 2013). For practical LIDAR intrinsic calibration, it is necessary to accurately know the extrinsic parameters of LIDAR and also completely eliminate the errors included in environmental modelling. However, when intrinsic calibration is conducted using actual LIDAR measurement, it is difficult to perfectly exclude the other error elements other than intrinsic errors. And it is also difficult to determine from which factor the error was corrected from the calibration result.

These problems can be overcome by using a LIDAR simulator. By using a LIDAR simulator, we know the true value of extrinsic parameters of the LIDAR. And since it is a virtual environment that we have constructed, errors caused by environment modelling can be excluded. Therefore, it is possible to completely exclude other error factors of the LIDAR and perform the intrinsic calibration. Also, errors can be inserted according to the error model of intrinsic parameters of the LIDAR, and the sizes of them are known. Through this, the calibration result can be verified by comparing the estimated intrinsic parameters with inserted errors.

In this study, plane-based calibration is applied as a method for intrinsic calibration of the LIDAR. The plane-based calibration is a calibration method using a plane that can be mathematically well-defined to estimate the orientation parameters of a LIDAR. (Glennie, Lichti, 2010) estimated intrinsic parameters using residuals from the plane, after scanning with a LIDAR in a place with several planes And (Lichti et al.2011) proposed a new error model for properly estimating intrinsic parameters that correlate with extrinsic parameters. In addition, many intrinsic calibration studies have been conducted mainly in indoor environments constructed with planes. (Lichti, 2007) and (Atanacio-Jiménez et al, 2011). When performing intrinsic calibration using planebased method in such environment, the accuracy of estimating varies according to various factors. Such factors include the number of scanning locations, the scan orientation to horizontal plane, the presence/absence of tilted scanning. Particularly, in plane-based intrinsic calibration conducted in a room structure constructed with planes, it is difficult to estimate the parameters of the LIDAR due to the correlation between a specific intrinsic parameter and an extrinsic parameter depending on the presence/absence of tilted observation. It is essential to evaluate the effect of such factor in order to properly verify the result of intrinsic calibration.

Therefore, in this study, the intrinsic calibration of the LIDAR according to the data acquisition method is verified using a 
LIDAR simulator. In the virtual environment of a room structure, the LIDAR simulation data including systematic errors of intrinsic parameters is generated. And the intrinsic parameters are estimated by applying the plane-based calibration. Finally, the possibility of estimating parameters and the correlation between parameters according to the data acquisition method are examined, and the results of estimate are analyzed.

\section{MATHEMATICAL MODEL}

A LIDAR is a sensor that acquires 3D geometric information by firing a laser pulse in a specific direction. The angle of the laser is defined as an angle in horizontal direction and an angle in vertical direction, and a $3 \mathrm{D}$ coordinates can be determined through a distance based on the origin. The coordinates of point cloud can be expressed in a spherical coordinate system using such angles and distances but can also be expressed in a 3D cartesian coordinate system. Equation (1) is an equation that converts the coordinates of point cloud expressed by the horizontal angle $(\varphi)$, vertical angle $(\theta)$ and distance $(D)$ into cartesian coordinates $(x, y, z)$, which does not include intrinsic parameters.

$$
P=\left[\begin{array}{l}
x \\
y \\
z
\end{array}\right]=R_{z}(\varphi) R_{y}(\theta)\left[\begin{array}{l}
D \\
0 \\
0
\end{array}\right]
$$

Typically, the intrinsic parameters of a LIDAR are defined as the offsets of the origin and the offsets of angles. The offsets of laser origin/angles mean the difference between the designed laser origin/angles and the actual ones. When the designed laser is fired in the $\mathrm{x}$-axis direction from the origin of the coordinate system, the origin and direction of the laser including each of offsets is shown in figure 1 and figure 2. Depending on the structure or mechanism of the LIDAR, the defined intrinsic parameter model is slightly different. In this study, a rotating LIDAR structure in which lasers aligned in a vertical direction and rotate in a horizontal direction is adopted and an intrinsic parameter model reflecting such structure is defined. The intrinsic parameter model in which the systematic error is inserted by including the offsets of origin and angles in the point cloud of equation (1) is defined as in equation (2).

$$
P=R_{z}(\varphi+\Delta \varphi) R_{y}(\theta+\Delta \theta)\left[\begin{array}{l}
D \\
0 \\
0
\end{array}\right]+R_{z}(\varphi+\Delta \varphi)\left[\begin{array}{l}
x_{0} \\
y_{0} \\
z_{0}
\end{array}\right]
$$

Where, $\Delta \varphi$ is the angular offset of horizontal rotation of the laser $\Delta \theta$ is the angular offset of vertical rotation of the laser $x_{0}, y_{0}$ and $z_{0}$ are the offsets of the laser origin, before horizontal rotation of the laser

In equation (2), the reason why the model contains only the rotation of $\mathrm{z}$-axis in the origin offset is because the aligned laser bundle of the rotating LIDAR rotates about the z-axis. Therefore, the offset of the origin is rotated about the $\mathrm{z}$-axis, which is defined by the above equation.

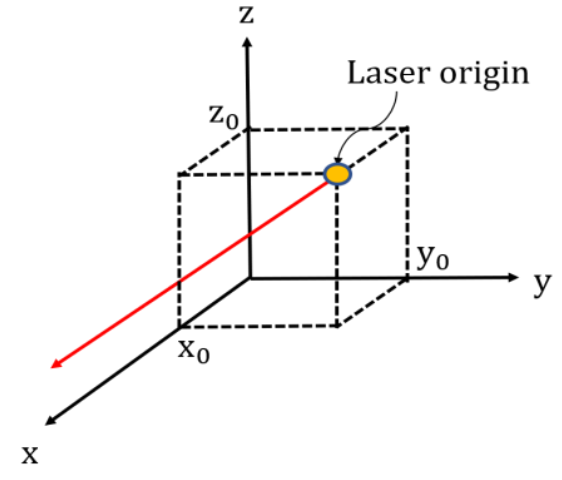

Figure 1. A laser with offset of laser origin

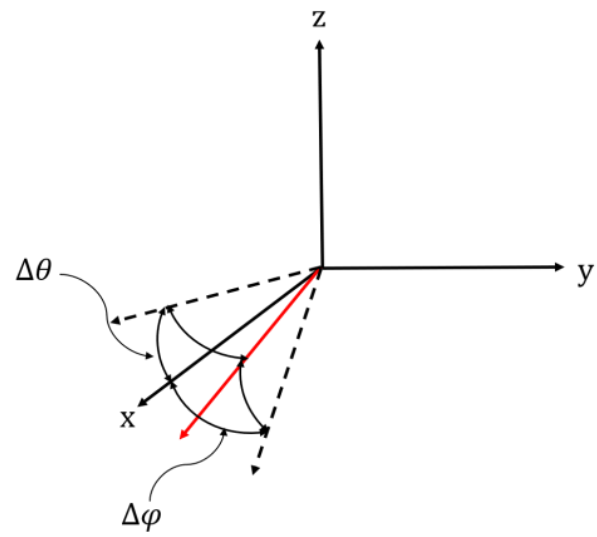

Figure 2. A laser with offsets of laser angles

\section{SIMULATED DATA}

\subsection{Simulated LIDAR}

In this study, a LIDAR simulator is constructed by stimulating VLP-16 from Velodyne, the most widely used commercial LIDAR. VLP-16 operates by rotating 16 laser bundles inside the LIDAR and scanning the surrounding environment. Our LIDAR simulator is designed by simulating the mechanism, laser direction, angular resolution of VLP-16 in the same way as the actual configuration of the LIDAR. The specification of the simulated LIDAR, VLP-16, is as follows.

\begin{tabular}{c}
\hline Velodyne VLP-16 Specification \\
\hline 16 Channels \\
Range: $100 \mathrm{~m}$ \\
Vertical FOV: $-15.0^{\circ}$ to $+15.0^{\circ}$ \\
Vertical Angular Resolution: $2.0^{\circ}$ \\
Horizontal FOV: $360^{\circ}$ \\
Horizontal Angular Resolution: $0.1^{\circ}-0.4^{\circ}$ \\
\hline
\end{tabular}

Table 1. Manufacturer specification of VLP-16 


\subsection{Simulated environment}

The plane-based calibration is mainly conducted in a place with planes or an indoor environment in a room structure. Accordingly, a virtual environment with a room structure of $10 \mathrm{~m} \times 10 \mathrm{~m} \times 5 \mathrm{~m}$ was constructed. When scanning in the middle of room structure, the data can have great symmetry, so in the room structure, the LIDAR was placed at a height of $1 \mathrm{~m}$ from the position expressed in figure 3 and scanned.

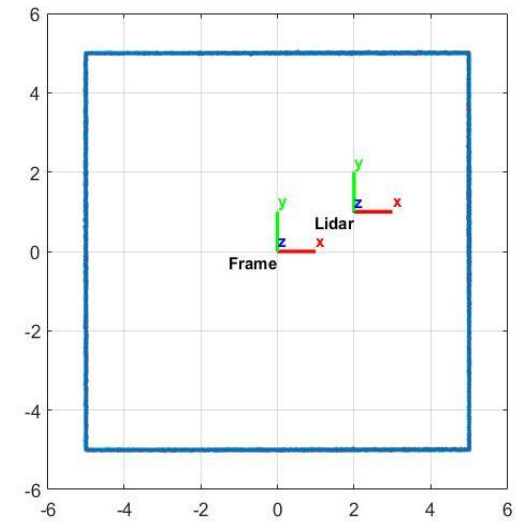

Figure 3. Scanning position of the LIDAR in the room

\subsection{Size of the offsets}

Since each of lasers has independent intrinsic parameters, values of intrinsic parameters are inserted differently in the 16 lasers of the simulator. For the offsets of vertical and horizontal angles, normal distribution random numbers with a standard deviation(5") were used. And normal distribution numbers with a standard deviation $(0.2 \mathrm{~cm})$ were generated for the offsets of laser origins. And random errors also exist in the acquired distances. Therefore, random errors are added to the acquired distances to generate LIDAR simulation data with noise.

\subsection{Observations}

Observations of a LIDAR include horizontal angle, vertical angle and distance. These observations are given as a result of scanning of a LIDAR, but the values of the intrinsic parameters contained are unknown. Thus, estimating the intrinsic parameters is the main purpose of the intrinsic calibration. In order to generate simulation data including the systematic error caused by intrinsic parameters, observations of horizontal and vertical angles are first generated. Since the horizontal and vertical angles have defined directions, angular observations can be generated according to configuration. The distances to the reflected points are calculated using the generated angular observations and intrinsic parameters. The distances are calculated by obtaining the distances $(D)$ of equation (2) that satisfies equation (3). Random errors are added to the calculated distances and final distance observations are generated.

$$
\vec{n} *(P-\vec{c})=0
$$
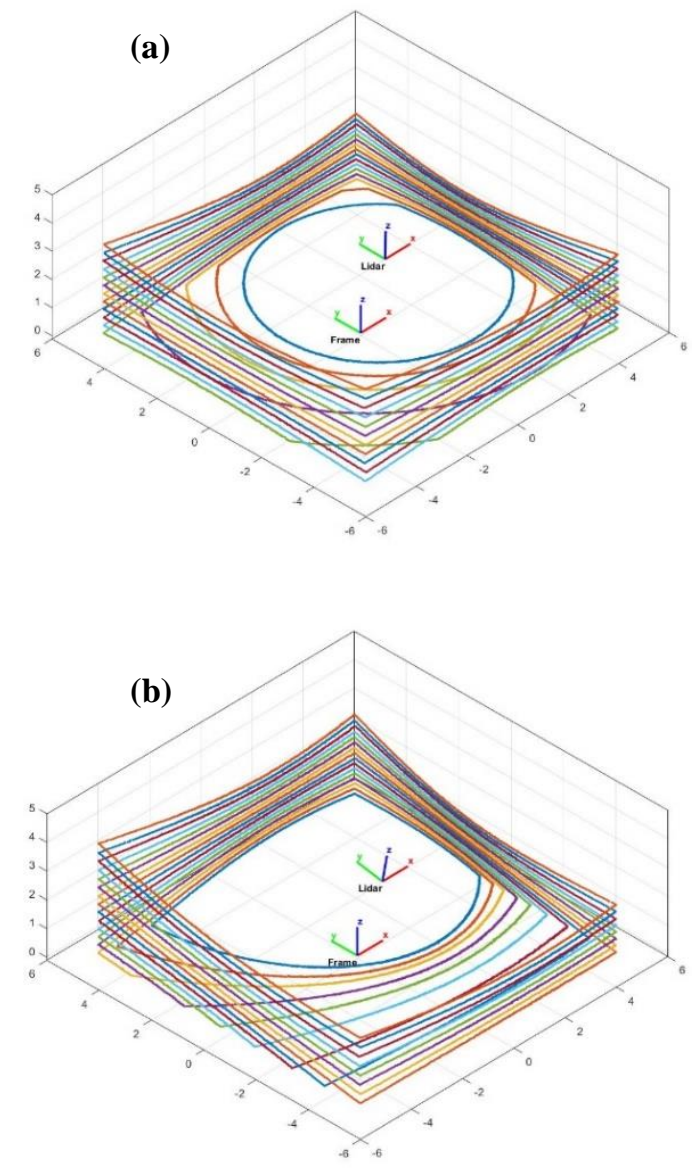

Figure 4. (a) Upright scanned LIDAR data (b) Tilted scanned LIDAR data

\subsection{Separation of scanning methods}

Method of LIDAR data acquisition is divided into two types. Basically, in a room-structured environment, a LIDAR can be installed straight on a tripod. The first method is a simulation of this type. A LIDAR is erected vertically on a horizontal floor in a virtual environment. The second method is scanning in a tilted orientation at the same position. A LIDAR is tilted $10^{\circ}$ in roll direction and scans the surroundings. Figure 4 shows the simulation data generated by scanning in two ways.

However, in the first method, if the LIDAR is set to be perfectly perpendicular to the horizontal floor, the observations are calculated regardless of a specific intrinsic parameter. Thus, it is impossible to estimate parameters using our intrinsic parameter model. The example of this problem is as follows. In figure 4 (a), when the laser pointing toward the bottom is called the $1^{\text {st }}$ laser, the $6^{\text {th }}$ to $16^{\text {th }}$ lasers scan only the side walls. If the LIDAR is installed perfectly vertically(parallel to the side walls) from the bottom plane, the observed distances are determined regardless of the intrinsic parameter $z_{0}$. Therefore, in order to estimate and analyze the parameters, scanning was conducted assuming that there was an inclination error about $1^{\circ}$ in the straightly installed LIDAR instead of perfectly installed way in the first method. 


\section{PLANE-BASED CALIBRATION}

The plane-based calibration is a method of scanning with a LIDAR in a place with several planes that can be mathematically well-defined, and estimating the parameters of a LIDAR using the constraint that the acquired point cloud exists on the planes. In this study, LIDAR simulation data including systematic error caused by intrinsic parameters is generated in a virtual environment with planar structures, and intrinsic parameters are estimated using this plane-based calibration.

The process of estimating intrinsic parameters using planebased calibration is as follows. The environment model and the extrinsic parameters of the LIDAR are given, and observations for the laser angles are also given. Thus, if the initial intrinsic parameters are set to 0 , the distances to the planes according to the laser angles are calculated through equation (2) and (3). When the calculated distance is called $D^{\prime}$, the equation for $D^{\prime}$ is expressed as equation (4). Since $D^{\prime}$ depends on the intrinsic parameters, it is expressed as a function of them.

$$
D^{\prime}=f\left(\Delta \varphi, \Delta \theta, x_{0}, y_{0}, z_{0}\right)
$$

Finally, we estimate the intrinsic parameters $\left(\Delta \varphi, \Delta \theta, x_{0}, y_{0}, z_{0}\right)$ which make the residuals to the minimum between the distance $D$ of LIDAR simulation data and $D^{\prime}$. In addition, since each of the 16 lasers has independent offsets of angles and origins, intrinsic parameters are estimated for each laser.

\section{ANALYSIS OF RESULTS}

Figure 5 shows the residuals calculated through the difference between all of the simulation points and the planes. In figure 5 , (a) and (b) are the residuals before and after the calibration with upright scanned data. And (c), (d) are those with tilted scanned data. Since the residuals before calibration contain the systematic errors caused by intrinsic parameters, it is observable that they have some pattern. Both scanning methods used the same intrinsic parameters to generate data and calculated residuals, but the tendency of the residuals differs depending on the presence/absence of tilt. After calibration, it seems that the patterns of residuals disappear.

\begin{tabular}{|c|c|}
\hline & Estimate Precision \\
\hline Upright & 0.009989 \\
\hline Tilted & 0.009962 \\
\hline
\end{tabular}

Table 1. Estimate precision of two type of scan

Table 3 shows the precision of estimate of two methods after estimating intrinsic parameters. Since the intrinsic parameters were estimated for each 16 lasers, the averaged statistical results were expressed. In both methods, the precisions of estimate are shown at a level similar to the inserted random errors. Through this, it can be considered that the intrinsic parameters are properly estimated and the systematic errors are properly removed.
Since the data was generated using the simulator, the true values of the intrinsic parameters are given. Thus, the difference between the estimated parameters and the true values of them can be also calculated. Figure 6 shows inserted and estimated intrinsic parameters of each laser according to the acquisition method. In figure 6 , the red graphs indicate the inserted intrinsic parameters, the black graphs in first row show the estimated intrinsic parameters from upright scanned data, and the black graph in second row shows those from tilted scanned data.

\begin{tabular}{|l|c|c|c|c|c|}
\hline \multicolumn{6}{|c|}{ RMSE of estimated parameters } \\
\hline & \multicolumn{2}{|c|}{ Unit: deg } & \multicolumn{3}{c|}{ Unit: $\mathrm{m}$} \\
\hline & $\Delta \varphi$ & $\Delta \theta$ & $x_{0}$ & $y_{0}$ & $z_{0}$ \\
\hline Upright & 0.0483 & 0.0783 & 0.0007 & 0.0027 & 0.0203 \\
\hline Tilted & 0.0163 & 0.0502 & 0.0005 & 0.0015 & 0.0050 \\
\hline
\end{tabular}

Table 2. RMSE of estimated intrinsic parameters of two scanning methods

Table 4 shows the result of calculating the RMSE of the estimated parameters of two scanning methods. In view of the residuals after estimate in figure 5 and the estimate precision in table 3, both methods seemed to have properly estimated parameters, but the results compared with the inserted valued were different. This is clearly seen in Table 4 . The RMSE calculated for the same parameters in the two methods differs up to 4 times. Particularly, in the upright method, the estimated $z_{0}$ was very in accurate.

And in the inserted and estimated parameters of the upright method in figure 6 , the differences from the true values show the same tendency among specific parameters. In the upright scanning, the estimated $\left(\Delta \varphi\right.$ and $\left.y_{0}\right)$ of the $1^{\text {st }}$ and $2^{\text {nd }}$ laser channels have a large difference from the inserted parameters. Also, it is noticeable that the estimated $\left(\Delta \theta\right.$ and $\left.z_{0}\right)$ in the 6 to 16 laser channels mainly scanning only the side walls show a considerable difference from the true value. This is because the correlations between the two parameters are large or it is not easy to estimate the corresponding parameters using given residuals.

\begin{tabular}{|c|l|l|l|l|l|}
\hline \multicolumn{6}{|c|}{ Correlation Coefficient } \\
\hline & \multicolumn{1}{|c|}{$\Delta \varphi$} & \multicolumn{1}{|c|}{$\Delta \theta$} & \multicolumn{1}{|c|}{$x_{0}$} & \multicolumn{1}{|c|}{$y_{0}$} & $z_{0}$ \\
\hline$\Delta \varphi$ & $\mathrm{x}$ & -0.019 & 0.030 & -0.991 & -0.019 \\
\hline$\Delta \theta$ & $\mathrm{x}$ & $\mathrm{x}$ & 0.127 & 0.024 & -0.657 \\
\hline$x_{0}$ & $\mathrm{x}$ & $\mathrm{x}$ & $\mathrm{x}$ & -0.025 & 0.183 \\
\hline$y_{0}$ & $\mathrm{x}$ & $\mathrm{x}$ & $\mathrm{x}$ & $\mathrm{x}$ & 0.018 \\
\hline$z_{0}$ & $\mathrm{x}$ & $\mathrm{x}$ & $\mathrm{x}$ & $\mathrm{x}$ & $\mathrm{x}$ \\
\hline
\end{tabular}

Table 3. Correlation coefficient of estimated parameters of upright scanned LIDAR data

\begin{tabular}{|c|l|l|l|l|l|}
\hline \multicolumn{6}{|c|}{ Correlation Coefficient } \\
\hline & \multicolumn{1}{|c|}{$\Delta \varphi$} & \multicolumn{1}{|c|}{$\Delta \theta$} & \multicolumn{1}{|c|}{$x_{0}$} & \multicolumn{1}{c|}{$y_{0}$} & $z_{0}$ \\
\hline$\Delta \varphi$ & $\mathrm{x}$ & -0.257 & -0.177 & -0.958 & 0.196 \\
\hline$\Delta \theta$ & $\mathrm{x}$ & $\mathrm{x}$ & 0.572 & 0.225 & -0.952 \\
\hline$x_{0}$ & $\mathrm{x}$ & $\mathrm{x}$ & $\mathrm{x}$ & 0.173 & -0.500 \\
\hline$y_{0}$ & $\mathrm{x}$ & $\mathrm{x}$ & $\mathrm{x}$ & $\mathrm{x}$ & -0.177 \\
\hline$z_{0}$ & $\mathrm{x}$ & $\mathrm{x}$ & $\mathrm{x}$ & $\mathrm{x}$ & $\mathrm{x}$ \\
\hline
\end{tabular}

Table 4. Correlation coefficient of estimated parameters of tilted scanned LIDAR data 

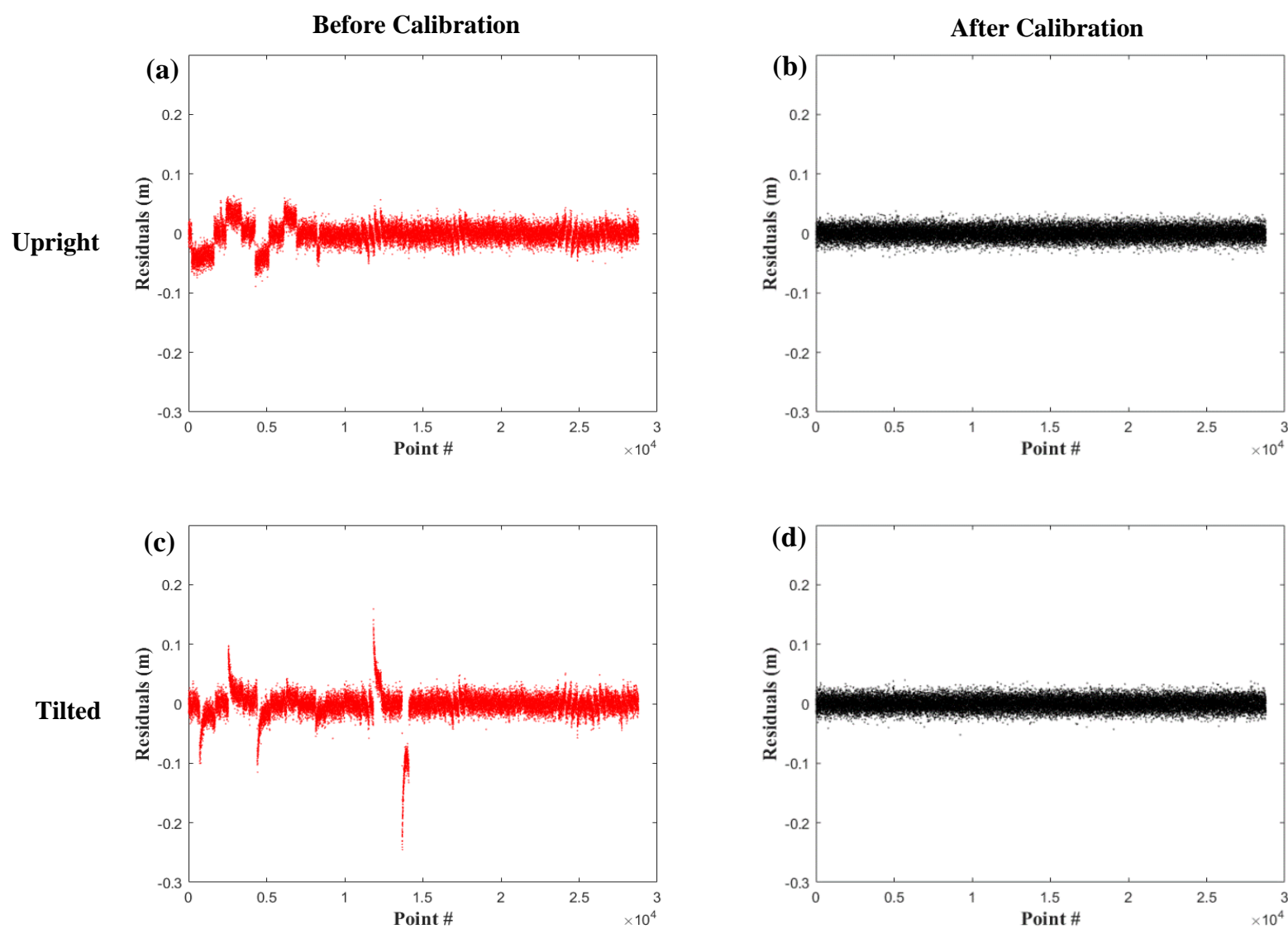

Figure 5. (a) Range residuals of upright scanned data before calibration

(b) Range residuals of upright scanned data after calibration

(c) Range residuals of tilted scanned data before calibration

(d) Range residuals of tilted scanned data after calibration
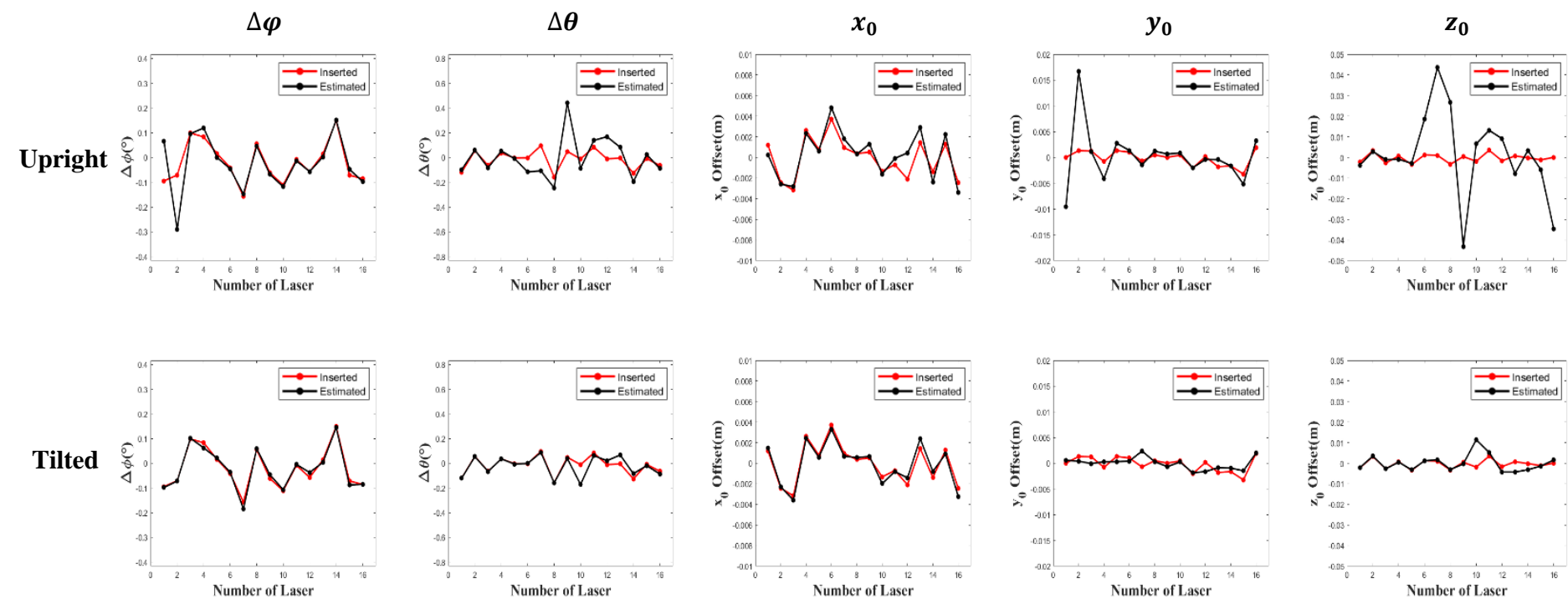

Figure 6. Inserted and estimated intrinsic parameters of each laser of two scanning methods 
Table 5 and 6 are tables that calculate the correlations between the estimated parameters of upright and tilted scanning. The estimated ( $\Delta \varphi, y_{0}$ ) in table 5 have a large correlation close to 1 . This shows that the reason $\left(\Delta \varphi, y_{0}\right)$ were not properly estimated in the upright method is due to the high correlation of them. In the table 6 , the correlation between the $\left(\Delta \varphi, y_{0}\right),\left(\Delta \theta, z_{0}\right)$ is relatively high. However, despite the correlation between parameters, it seems that all parameters are properly estimated because the laser scans multiple planes and is incident at various angles due to the tilting effect.

\section{CONCLUSION}

In this study, the results of the intrinsic calibration according to the presence/absence of the tilt in a room structure environment were analyzed and verified by using a LIDAR simulator. A LIDAR simulator was designed according to the mechanism of a commercial LIDAR, and a mathematical model with intrinsic parameters suitable for the corresponding LIDAR mechanism was defined. In addition, for the plane-based calibration, a virtual environment of a room structure was constructed, and LIDAR simulation data in which systematic errors were inserted by intrinsic parameters was generated in the environment. Since the simulator was used, other error factors were excluded, whereby the intrinsic parameters of the LIDAR could be estimated using the residuals caused only by the intrinsic systematic errors and the distance random errors. Additionally, since the inserted parameters are known, verification and analysis of the corresponding algorithm can be properly done through comparison with the estimated parameters.

In our study, only the tilting effect of scanning was evaluated among the factors affecting the results of the intrinsic calibration. When considering too many factors, it is difficult to infer which factors influenced the results of calibration, so other factors were fixed. By fixing these other factors, the result of this study may be applicable only in certain situation. Thus, if the analysis of the influence of each factor is performed in future research, a more general verification of intrinsic calibration can be conducted.

\section{ACKNOWLEDGEMENT}

This research supported by the Institute of Information \& Communication Technology Planning \& Evaluation(IITP) funded by the Ministry of Science, ICT \& Future Planning. (No. 2019-0-00399, Development of SW technology for recognition, judgement and path control algorithm verification simulation and dataset generation)

\section{REFERENCES}

Atanacio-Jiménez, G., González-Barbosa, J. J., HurtadoRamos, J. B., Ornelas-Rodríguez, F. J., Jiménez-Hernández, H., García-Ramirez, T., González-Barbosa, R., 2011. Lidar velodyne hdl-64e calibration using pattern planes. International Journal of Advanced Robotic Systems, 8(5), 59.
Chow, J. C., Lichti, D. D., Glennie, C., Hartzell, P., 2013. Improvements to and comparison of static terrestrial LiDAR selfcalibration methods. Sensors, 13(6), 7224-7249.

Glennie, C., \& Lichti, D. D., 2010. Static calibration and analysis of the Velodyne HDL-64E S2 for high accuracy mobile scanning. Remote sensing, 2(6), 1610-1624.

Lichti, D. D., 2007. Error modelling, calibration and analysis of an AM-CW terrestrial laser scanner system. ISPRS journal of photogrammetry and remote sensing, 61(5), 307-324.

Lichti, D. D., 2010. Terrestrial laser scanner self-calibration: Correlation sources and their mitigation. ISPRS Journal of Photogrammetry and Remote Sensing, 65(1)

Lichti, D. D., Chow, J., \& Lahamy, H., 2011. Parameter decorrelation and model-identification in hybrid-style terrestrial laser scanner self-calibration. ISPRS Journal of Photogrammetry and Remote Sensing, 66(3), 317-326.

Muhammad, N., \& Lacroix, S., 2010. Calibration of a rotating multi-beam lidar. In 2010 IEEE/RSJ International Conference on Intelligent Robots and Systems (pp. 5648-5653). IEEE.

Skaloud, J., \& Lichti, D., 2006. Rigorous approach to boresight self-calibration in airborne laser scanning. ISPRS journal of photogrammetry and remote sensing, 61(1), 47-59. 